\title{
3DSAL: An Efficient 3D-CNN Architecture for Video Saliency Prediction
}

\author{
Yasser Abdelaziz Dahou Djilali ${ }^{1,3}$, Mohamed Sayah ${ }^{2}$, Kevin McGuinness ${ }^{3}$ Noel E. O'Connor ${ }^{3}$ \\ ${ }^{1}$ Institut National des Télécommunications et des TIC, Oran, Algeria \\ ${ }^{2}$ Université Oran1, FSEA, Oran, Algeria, \\ sayah.mohamed@univ-oran1.dz. \\ ${ }^{3}$ Insight Center for Data Analytics, Dublin City University, Dublin 9, Ireland, \\ noel.oconnor@insight-centre.org
}

\begin{abstract}
Keywords: Visual attention, Video saliency, Deep learning, 3D CNN.
Abstract: In this paper, we propose a novel 3D CNN architecture that enables us to train an effective video saliency prediction model. The model is designed to capture important motion information using multiple adjacent frames. Our model performs a cubic convolution on a set of consecutive frames to extract spatio-temporal features. This enables us to predict the saliency map for any given frame using past frames. We comprehensively investigate the performance of our model with respect to state-of-the-art video saliency models. Experimental results on three large-scale datasets, DHF1K, UCF-SPORTS and DAVIS, demonstrate the competitiveness of our approach.
\end{abstract}

\section{INTRODUCTION}

Human attention was considered first in philosophy, later in psychology and neuroscience, and most recently as a computer vision problem in the field computer science (Mancas et al., 2016). Thanks mainly to advances in deep learning, the development of computational models of human attention has received renewed research interest in recent years (Mancas et al., 2016). Computational models have been proposed for imitating the attentional mechanisms of the Human Visual Systems (HVS) for both dynamic and static scenes. Dynamic fixation prediction, or video saliency prediction, is very useful for understanding human attentional behaviors for video content and has multiple practical real-world applications e.g. video captioning, compression, question answering, object segmentation (Wang et al., 2018). It is thus highly desirable to have robust, high performance video saliency prediction models.

Recently introduced benchmarks such as DHF1K (Wang et al., 2018) and LEDOV (Jiang et al., 2018a), have allowed researchers to effectively train deep learning models in an end-to-end manner by formulating saliency as a regression problem. However, the reported performances on video (dynamic scene) datasets according to commonly used saliency metrics are still far from those reported for images (static scene). This is most likely due to the rapid transition of video frames that makes dynamic saliency prediction very challenging. Latent video saliency deep learning based models separate spatial features from temporal features. This is implemented, for example, by a CNN module to extract spatial features, which can then be aggregated into an LSTM module to capture the temporal features (Hochreiter and Schmidhuber, 1997).

In this paper we propose a novel video saliency model that uses a 3D CNN architecture (Ji et al., 2013). When performing a cubic convolution, our model captures spatio-temporal features in one 3D CNN module. The dimensions $r$ and $s$ of the cube extract the spatial features while the $t$ axis extracts the temporal features. In this way, the model learns saliency by fusing spatio-temporal features for calculating the final saliency map. Our key contribution is a 3D CNN architecture for predicting human gaze in dynamic scenes, which explicitly learns the hidden relationship between adjacent frames for accurate saliency prediction.

This paper is organized as follows: Section 2 provides an overview of related video saliency works. Section 3 gives a detailed description of the proposed deep saliency framework. Section 4 compares the experimental results to state-of-the-art methods. Finally, we conclude this work in Section 5. The results can be reproduced with the source code and trained models 
available via GitHub: Please find here.

\section{RELATED WORKS}

In this section we review the relevant literature on saliency models for both static and dynamic scenes.

\subsection{Static Saliency models}

Saliency prediction for images has been widely studied during the last few decades. As a pioneer, (Itti et al., 1998) derived bottom-up visual saliency using center-surround differences across multi-scale image features. Other static saliency models e.g. (Bruce and Tsotsos, 2006), (Garcia-Diaz et al., 2009), (Seo and Milanfar, 2009), (Goferman et al., 2012), (Gao and Vasconcelos, 2005) are mostly based on computing multiple visual features such as color, edge, and orientation at multiple spatial scales to produce a saliency map. More recent deep learning based static saliency models e.g. (Huang et al., 2015), (Kümmerer et al., 2014), (Liu et al., 2015), (Kruthiventi et al., 2017), (Cornia et al., 2016), (Pan et al., 2017) have achieved remarkable improvements relying on the success of neural networks and the availability of large-scale saliency datasets for static scenes, such as those described in (Bylinskii et al., ), (Jiang et al., 2015), (Borji and Itti, 2015).

(Vig et al., 2014) and (Kruthiventi et al., 2017) were the first to use CNNs for saliency prediction when introducing eDN and DeepFix respectively. DeepFix initialized the first 5 convolution blocks with VGG-16 weights, then added two novel Location Based Convolutional (LBC) layers to capture semantics at multiple scales. Pan et al. (Pan et al., 2017) utilized Generative Adversarial Networks (GANs) (Goodfellow et al., 2014) to build the SalGAN model. The network consists of a generator model whose weights are learned by backpropagation computed from a binary cross entropy (BCE) loss over existing saliency maps. The resulting prediction is processed by a discriminator network trained to solve a binary classification task between the saliency maps generated by the generative stage and the ground truth ones.

\subsection{Dynamic Saliency models}

Visual information constantly changes due to egocentric movements or the dynamics of the scene being observed. Dynamic Saliency is then dependent on both current scene saliency as well as the accumulated knowledge from previous time instants (Borji and Itti, 2013). Video saliency prediction is extremely more challenging than image saliency prediction (Borji, 2018). In this task, viewers have much less time to explore each video frame ( $\sim 1 / 30$ seconds) compared to the 3-5 seconds typical for viewing still images.

Early video saliency models such as (Leifman et al., 2017), (Garcia-Diaz et al., 2012), (Zhang and Sclaroff, 2013), (Leboran et al., 2017), (Xu et al., 2017), (Guo et al., 2008), (Rudoy et al., 2013), rely on existing static saliency models with additional motion features. They generally use linear or nonlinear combination rules to fuse spatial and temporal information. However, using a simple fixed weight to combine spatial and temporal information can drive the model to lose the intrinsic relationship between these two complementary aspects.

Few works have investigated deep learning based video saliency prediction models ( (Bak et al., 2018), (Jiang et al., 2017), (Wang et al., 2018), (Adel Bargal et al., 2018)). They are mainly based on two distinct network modules to deal with spatial and temporal fields separately. These works exhibit strong performance and show the potential of using neural networks to the video saliency problem. (Bak et al., 2018) were the first to leverage deep learning when they used a two-stream CNN architecture for video saliency prediction. Video frames and motion maps were fed to the two streams. (Wang et al., 2018), proposed a CNN-LSTM network architecture with an attention mechanism to explicitly encode static saliency information, thus allowing the LSTM to focus on learning a more flexible temporal saliency representation across successive frames. (Panagiotis Linardos, 2018) introduced a temporal regularization for their previous model SalGAN (Pan et al., 2017) for static saliency prediction. In terms of architecture, they added a convolutional LSTM layer on top of the frame-based saliency prediction to adapt it for dynamic saliency prediction.

All these works consider the spatial domain as the most influential aspect, and use very little accumulated knowledge from the past $(\sim 70 \mathrm{~ms})$, while the average human eye reaction time, is of the order of $284 m s$ (Saslow, 1967). We propose that more importance needs to be given to the temporal domain for video saliency prediction. In our approach, we exploit the temporal and spatial domain in an equal manner via the use of 3D CNN, with a view to more appropriate spatio-temporal feature learning (see Fig 1 . Fur- 
thermore, we smooth adjacent frames to obtain good eye fixation quality saturation by introducing the tangent hyperbolic weighting function on the input sequence frames.

\section{Our Approach}

\subsection{Overview}

Fig 2 represents the overall architecture of our video saliency model. The framework takes $i$ consecutive input frames: $\left\{F_{t-i}\right\} \in \mathrm{R}^{224 \times 244 \times 3}$ and where $i \in\{0, \ldots, 5\}$. Since the latency for sacadic eye movement is about 284ms (Saslow, 1967), when watching a $30 \mathrm{fps}$ video, the human eye is sensitive to exactly $(0.284 m s \times 30 \mathrm{fps}) \simeq 8$ frames. Thus, we set the number of input frames to six to approximate the ideal threshold and save computing capacity at the same time. Each frame is fed into the spatial feature extraction function modeled with pre-trained $V G G-16$ (Simonyan and Zisserman, 2014) after deleting the fully connected layers. This choice was motivated by the reduction of complexity while improving accuracy. Since $V G G-16$ was trained on large-scale Imagenet dataset (1.4M images) (Deng et al., 2009), $V G G-16$ provides excellent feature extraction performance. The output of the spatial feature extraction function is six feature cuboids: $F_{t-5}^{*}, F_{t-4}^{*}, \ldots ., F_{t}^{*} \in$ $\mathrm{R}^{14 \times 14 \times 512}$

$$
G\left(F_{x}\right)=F_{x}^{*} .
$$

and where the function $G$ is modelled by the truncated $V G G$-16 algorithm (- for more details see (Simonyan and Zisserman, 2014)).

\subsection{Weighting cuboid coefficients}

The eye motion on a given object has been intuitively considered as a tangent hyperbolic activation function (Nwankpa et al., 2018). To preserve the consistency of human vision and avoid computing complexity in time and space, we considered six (06) frames to define the motion features for the human eyes. This enables us to use a suitable tanh function, to weight the

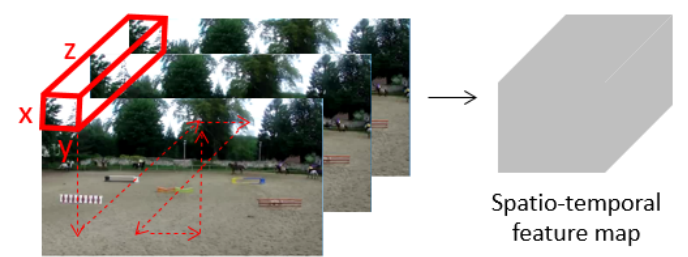

Figure 1: 3D Convolution operation over adjacent frames motion frames by parameters $c[i]$ where $i \in\{1, \ldots, 6\}$ - see bold weights in the TABLE 1 below.

Table 1: Fixation quality motion

\begin{tabular}{|c|c|c|c|}
\hline Weight & $\begin{array}{c}\text { Fixation } \\
\text { Quality } \\
\tanh (\mathrm{c}[\mathrm{i}])\end{array}$ & $\begin{array}{c}\text { Quality } \\
\text { Variation } \\
\tanh (\mathrm{c}[\mathrm{i}+1]) \\
-\tanh (\mathrm{c}[\mathrm{i}])\end{array}$ & $\begin{array}{c}\text { Quality } \\
\text { Saturation } \\
\tanh (\mathrm{c}[\mathrm{i}+1]) \\
+\tanh (\mathrm{c}[\mathrm{i}])\end{array}$ \\
\hline 0.1 & 0,044 & - & - \\
\hline 0.2 & 0,088 & 0.044 & 0.13 \\
\hline 0.3 & 0,134 & 0.046 & 0.22 \\
\hline $\mathbf{0 . 4}$ & 0.184 & 0.050 & $\mathbf{0 . 3 2}$ \\
\hline $\mathbf{0 . 5}$ & 0.239 & 0.055 & $\mathbf{0 . 4 2}$ \\
\hline $\mathbf{0 . 6}$ & 0.301 & 0.062 & $\mathbf{0 . 5 4}$ \\
\hline $\mathbf{0 . 7}$ & 0.377 & 0.076 & $\mathbf{0 . 6 8}$ \\
\hline $\mathbf{0 . 8}$ & 0.477 & 0.100 & $\mathbf{0 . 8 5}$ \\
\hline $\mathbf{0 . 9 8}$ & 0.998 & 0.521 & $\mathbf{1 . 4 7}$ \\
\hline- & 0,998 & 0,0 & $\mathbf{2 . 0 0}$ \\
\hline
\end{tabular}

Considering the vector parameters $c=$ $[0.4,0.5,0.6,0.7,0.8,0.98]$ as illustrated above, we can reach the eye fixation quality saturation in the $6^{\text {th }}$ spatial frame. As such, the temporal dimension is defined as a continuation of six $c$-weighted consecutive frames. In each iteration, the $6^{\text {th }}$ spatial frame is determined via an adversarial process in which the spatial frames $F_{t-5}, F_{t-4}, F_{t-3}, F_{t-2}$, and $F_{t-1}$ are used to compute the $F_{t}$ saliency map. Finally, each of the six cuboids, weighted with respect to their importance in the learning process, are adjusted in a temporary order to construct one spatio-temporal feature map. It should be noted that the six frames do not represent a large variation of space and that their concatenation preserves the spatial information when finding the correlation in time for each pixel location. Later, a spatio-temporal feature map of $6 \times$ frames determines the spatio-temporal units $\mathbb{S}$ $\in \mathrm{R}^{6 \times 14 \times 14 \times 512}$. The $3 \mathrm{D} C \mathrm{CNN}$ takes $\mathbb{S}$ as an input, to perform a spatio-temporal fusion, in order to learn saliency. Then, a saliency map $P \in \mathrm{R}^{224 \times 224}$ is generated to represent the saliency map for $F_{t}$.

\subsection{D CNN Architecture}

We believe that a 3D ConvNet is more appropriate for spatio-temporal feature learning. Compared to a 2D ConvNet, a 3D ConvNet has the ability to model temporal information better by virtue of $3 \mathrm{D}$ convolutions and 3D pooling operations. In 2D ConvNets, convolution and pooling operations are only performed spatially. 3D ConvNets perform those operations spatio-temporally to conserve the temporal information of the input signals resulting in an output volume (see Fig 1). The same phenomena 


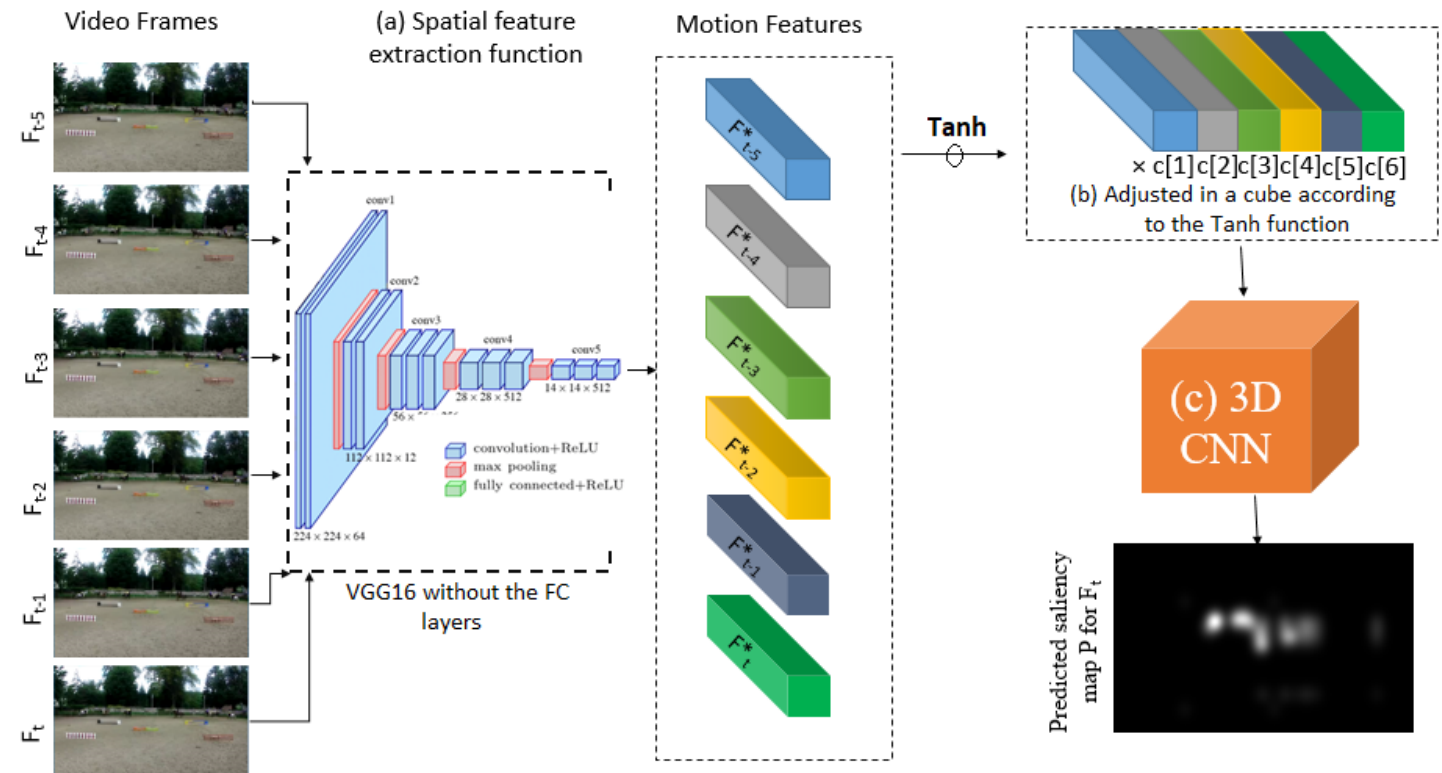

Figure 2: Network Architecture of 3DSAL. (a) Feature extraction function with the truncated VGG-16. (b) Composition of adjacent feature maps via the use of Tanh activation function. (c) Spatio-temporal feature fusion with 3D CNN.

is applicable for 2D and 3D pooling. (Tran et al., 2015).

As shown in TABLE 2, we built a five block decoder 3D CNN network to learn saliency in a slow fusion manner. Each block consists of Deconv3D and operations, with a Relu (Rectified Linear Unites) activation function. The role of Deconv3D is to up-sample the feature map resolution and Conv3D to construct the spatio-temporal features for saliency learning. Also, we denote the triplet $(t, r, s)$ as the kernel size for Deconv3D and Conv3D layers. Further, we use the $t$ axis in the kernel to cover the temporal dimension in the kernel, while, $(r, s)$ denotes the spatial kernel size. Let us consider a convolutional layer $l$ and the input spatio-temporal units $\mathbb{S}$. The $i^{\text {th }}$ output unit $V_{i, K}{ }^{(l)}$ for the layer $l$ is computed by using the equation (2).

$$
\begin{gathered}
\left(\begin{array}{c}
V_{i, K}^{(l)} \\
(x, y, z)
\end{array}\right)=\sum_{c=1}^{C}\left(\sum_{t=1}^{T} \sum_{r=1}^{R} \sum_{s=1}^{S}\left(\begin{array}{l}
W_{i, K, c}^{(l)} \times \underbrace{(l-1)}_{(t, r, s)} \\
U_{i, c}^{(x+t, y+r, z+s)}
\end{array}\right)\right) \\
+\left(B_{i, K}^{(l)}\right) .
\end{gathered}
$$

where $C$ is the channel number for the layer ( $l$ ) and $x, y, z$ are the cubic spatial dimensions respectively. The parameter $K$ is considered as the channel dimension for the output unit $V^{(l)}$. The
$W_{i, K, c}^{(l)}$ term denotes the weights connecting the $i^{t h}$ unit at position $(x, y, z)$ in the feature map of layer $(l-1)$ and the $i^{t h}$ unit in the layer $l$ with $K$ channels. Finally, the $B_{\forall i, K}^{(l)}$ term is the bias vector with length $K$.

The authors of (Li et al., 2016) and (Tran et al., 2015) demonstrated that the most suitable kernel size for $3 \mathrm{D}$ convolution is $3 \times 3 \times 3$. Hence, we set the $3 \mathrm{D}$ convolution kernel size to $3 \times 3 \times 3$ with stride $1 \times 1 \times 1$. Since the ground truth saliency map can be seen as a distribution probability, where each pixel represents the probability to be fixated by a human, at the final block, we use Sigmoid as an activation function to get a normalized predicted saliency map in $[0,1]$ with a size $224 \times 224$.

Loss function. The saliency loss is computed on a per-pixel basis, where each value of the predicted saliency map is compared with its corresponding peer from the ground truth map. We denote the predicted saliency map as $P \in[0,1]^{224 \times 224}$ and the continuous saliency map as $G \in[0,1]^{224 \times 224}$. The continuous saliency map $G$ is obtained by blurring the binary fixation map $F M$ with a $2 \mathrm{D}$ Gaussian kernel. The fixation map $F M$ is a binary image with:

$$
F M_{i j}= \begin{cases}1 & \text { if location }(i, j) \text { is a fixation } \\ 0 & \text { otherwise }\end{cases}
$$

and the variance of the Gaussian is selected so the filter covers approximately 1-degree of visual angle, as 
Table 2: Architecture of the 3D CNN

\begin{tabular}{|c|l|l|c|c|l|}
\hline Layer & Depth & $\begin{array}{l}\text { Kernel } \\
\text { /Pool }\end{array}$ & $\begin{array}{c}\text { Output } \\
\text { Shape }\end{array}$ & $\begin{array}{c}\text { Params } \\
\#\end{array}$ & Act \\
\hline Conv3D_1_1 & 512 & $3 \times 3 \times 3$ & $(6,14,14,512)$ & 7078400 & Relu \\
Conv3D_1_2 & 512 & $3 \times 3 \times 3$ & $(6,14,14,512)$ & 7078400 & Relu \\
MaxPool3D_1 & - & $4 \times 2 \times 2$ & $(3,7,7,512)$ & 0 & - \\
Batch-Norm & - & - & $(3,7,7,512)$ & 2048 & - \\
\hline Deconv3D_1 & 512 & $1 \times 3 \times 3$ & $(3,14,14,512)$ & 2359808 & Relu \\
Conv3D_2_1 & 512 & $3 \times 3 \times 3$ & $(3,14,14,512)$ & 7078400 & Relu \\
Batch-Norm & - & - & $(3,7,7,512)$ & 2048 & - \\
\hline Deconv3D_2 & 256 & $3 \times 3 \times 3$ & $(3,28,28,256)$ & 3539200 & Relu \\
Conv3D_2_1 & 256 & $3 \times 3 \times 3$ & $(3,28,28,256)$ & 179728 & Relu \\
Conv3D_2_2 & 256 & $3 \times 3 \times 3$ & $(3,28,28,256)$ & 179728 & Relu \\
MaxPool3D_2 & - & $3 \times 1 \times 1$ & $(1,28,28,256)$ & 0 & - \\
Batch-Norm & - & - & $(1,28,28,256)$ & 1024 & - \\
\hline Deconv3D_3 & 128 & $1 \times 3 \times 3$ & $(1,56,56,128)$ & 295040 & Relu \\
Conv3D_3_1 & 128 & $1 \times 3 \times 3$ & $(1,56,56,128)$ & 147584 & Relu \\
Conv3D_3_2 & 128 & $1 \times 3 \times 3$ & $(1,56,56,128)$ & 147584 & Relu \\
Batch-Norm & - & - & $(1,56,56,128)$ & 512 & - \\
\hline \hline Deconv3D_4 & 64 & $1 \times 3 \times 3$ & $(1,112,112,64)$ & 73792 & Relu \\
Conv3D_4_1 & 64 & $1 \times 3 \times 3$ & $(1,112,112,64)$ & 36928 & Relu \\
Conv3D_4_2 & 64 & $1 \times 3 \times 3$ & $(1,112,112,64)$ & 36928 & Relu \\
Batch-Norm & - & - & $(1,112,112,64)$ & 2048 & - \\
\hline Deconv3D_5 & 32 & $1 \times 3 \times 3$ & $(1,224,224,32)$ & 18464 & Relu \\
Conv3D_5_1 & 32 & $1 \times 3 \times 3$ & $(1,224,224,32)$ & 9258 & Relu \\
Conv3D_5_2 & 16 & $1 \times 3 \times 3$ & $(1,224,224,16)$ & 4624 & Relu \\
Conv3D_5_3 & 1 & $1 \times 3 \times 3$ & $(1,224,224,1)$ & 145 & Sigm \\
\hline & & Total Params: $31,447,841$ & & \\
\hline
\end{tabular}

done in (Judd et al., 2012).

The saliency task can be seen as a similarity measure between the predicted saliency map $P$ and the ground truth $G$. The loss function must be designed to maximise the invariance of predictive maps and give higher weights to locations with higher fixation probability. An appropriate loss for this situation is the binary cross entropy, defined as:

$$
\mathcal{L}_{B C E}(G, P)=-\frac{1}{N} \sum_{i=1}^{N}\left(G_{i} \log \left(P_{i}\right)+\left(1-G_{i}\right) \log \left(1-P_{i}\right)\right)
$$

\section{Experiments}

\subsection{Experimental setup}

Datasets. DHF1K (Wang et al., 2018), LEDOV (Jiang et al., 2018b), HOLYWOOD (Mathe and Sminchisescu, 2015), UFC-SPORT (Mathe and Sminchisescu, 2015) and DIEM (Mital et al., 2011) are the five datasets widely used for video saliency research. DHF1K comprises a total of 1,000 video sequences with 582,605 frames covering a wide range of scenes, motions and activities. HOLLYWOOD-2 is a dynamic eye tracking dataset. It contains short video sequences from a set of 69 Hollywood movies, containing 12 different human action classes, ranging from answering a phone, eating, driving and running. The UCF-Sports dataset consists of 150 videos covering 9 sports classes like golf, skateboarding, running and riding. LEDOV contains videos with a total of 179,336 frames covering three main sub-categories: Animals, Man-made-Objects and Human activities varying from social activities, daily actions, sports and art performance.

We have chosen DHF1K and UFC-SPORT to train our 3DSAL model. DHF1K characterises the free viewing approach, in which subjects freely watch the stimuli so that many internal cognitive tasks are engaged, thereby making the generated saliency map more difficult to predict. UFC-SPORT is a task driven dataset, where subjects are more likely to follow the main objects in the scene, affording the model precision. Training on two different paradigms, helps ensure more robust prediction.

Training protocol. We have two training modes: (1) 3DSAL-base: Training the model without regression, where all frames are fed into the $3 \mathrm{D} \mathrm{CNN}$ in an equal manner, without multiplying by the weighting coefficients. (2) 3DSAL-weighted: The use of weighting coefficients, to indicate the frame importance in the prediction process.

For DHF1K, we respect the original training/validation/testing partitioning (600/100/300). For UFC-SPORT, as proposed by the authors in (Mathe and Sminchisescu, 2015), the training/testing is split (103/47). We test our model on: DHF1K, UFC-SPORT and DAVIS (Perazzi et al., 2016) for both quantitative and qualitative results.

Technical specification. We implemented our model in Python using the Keras API running a TensorFlow backend. Due to the huge size of the training data (550k frames), we used the early stopping technique on the validation set for optimal generalization performance (Prechelt, 1998). The Adam Optimizer (Kingma and Ba, 2014) initial learning rate was set to $10^{-4}$ and was dropped by 10 each 2 epochs. The network was trained for 33 epochs. The entire training procedure took about 7 days (160 hours) with a single NVIDIA GTX 1080 GPU,which has a total amount of 8 GBytes global memory and has 20 multiprocessors, and i7 $7820 \mathrm{HK}$ 3.9GHZ Intel processor.

Metrics. To test the performance of our model, we utilize the five widely used metrics: AUC-Judd (AUC-J), Similarity metric (SIM), Linear Correlation Coefficient (CC), shuffled AUC (s-AUC) and 


\begin{tabular}{|c|c|c|c|c|c|c|c|c|c|c|}
\hline \multirow[t]{2}{*}{ Dataset } & \multicolumn{5}{|c|}{ DHF1K } & \multicolumn{5}{|c|}{ UFC-SPORT } \\
\hline & AUC-J $\uparrow$ & $\operatorname{SIM} \uparrow$ & $\mathrm{s}-\mathrm{AUC} \uparrow$ & $\mathrm{CC} \uparrow$ & $\mathrm{NSS} \uparrow$ & AUC-J $\uparrow$ & $\mathrm{SIM} \uparrow$ & $\mathrm{s}-\mathrm{AUC} \uparrow$ & $\mathrm{CC} \uparrow$ & $\mathrm{NSS} \uparrow$ \\
\hline \# OBDL (Hossein Khatoonabadi et al., 2015) & 0.638 & 0.171 & 0.500 & 0.117 & 0.495 & 0.759 & 0.193 & 0.634 & 0.234 & 1.382 \\
\hline \# AWS-D Leboran et al., 20IT) & 0.703 & 0.157 & 0.513 & 0.174 & 0.940 & 0.823 & 0.228 & 0.750 & 0.306 & 1.631 \\
\hline OM-CNN Jiang et al., 2017) & 0.856 & 0.256 & 0.583 & 0.344 & 1.911 & 0.870 & 0.321 & 0.691 & 0.405 & 2.089 \\
\hline Two-Stream Bak et al., 2018 & 0.834 & 0.197 & 0.581 & 0.325 & 1.632 & 0.832 & 0.264 & 0.685 & 0.343 & 1.753 \\
\hline ACLNET (Wang et al., 2018 & 0.890 & 0.315 & 0.601 & 0.434 & 2.354 & 0.897 & 0.406 & 0.744 & 0.510 & 2.567 \\
\hline Linardos et al Panagiotis Linardos, 2018. & 0.744 & 0.260 & 0.722 & 0.302 & 2.246 & - & - & - & - & - \\
\hline 3DSAL-Base & - & - & - & - & - & 0.8111 & 0.3255 & 0.6088 & 0.3209 & 1.7119 \\
\hline 3DSAL-Weighted & 0.8500 & 0.3205 & 0.6234 & 0.3562 & 1.9962 & 0.8813 & 0.4783 & 0.7011 & 0.5902 & 2.8023 \\
\hline
\end{tabular}

Table 3: Comparative performance study on: DHF1K, UFC-SPORT datasets.
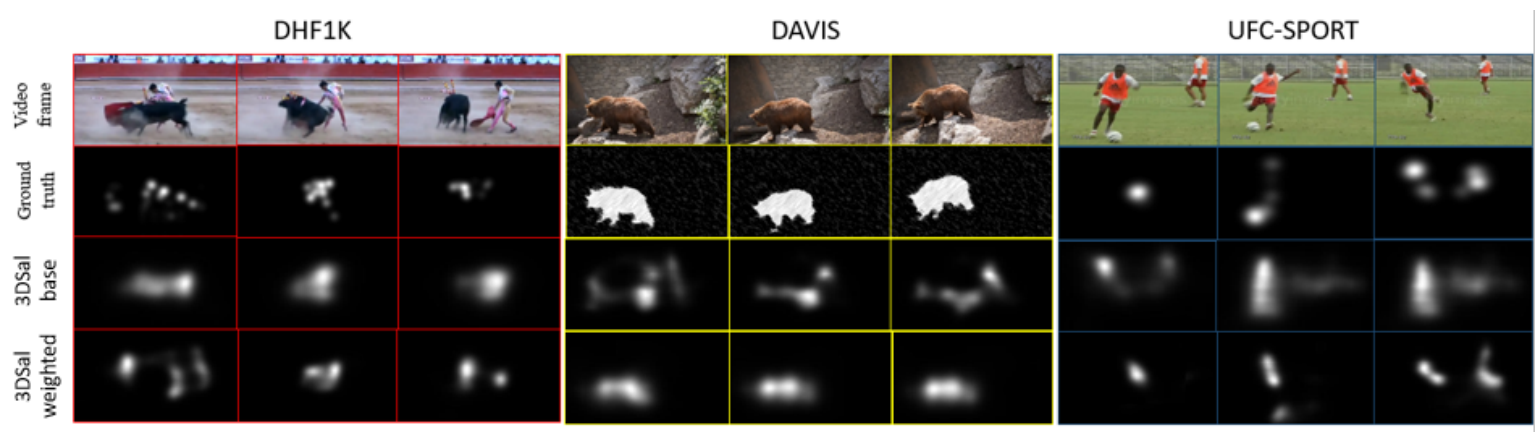

Figure 3: Saliency map predictions over three datasets.

Normalized Scanpath Saliency (NSS). A detailed description of these metrics is presented in (Borji and Itti, 2013).

Competitors. We compare the performance of our model according to the different saliency metrics, with six video saliency models: OM-CNN (Jiang et al., 2017), Two-stream (Bak et al., 2018), AWSD (Leboran et al., 2017), OBDL (Hossein Khatoonabadi et al., 2015), ACLNet (Wang et al., 2018), (Panagiotis Linardos, 2018). Benefiting from the work of (Wang et al., 2018), which tested the performance of the previous models in three Datasets: DHF1K, HOLYWOOD-2, UFC-SPORT. We add our results to this work, in order, to compare the performance of our model with the aforementioned works.

\subsection{Results}

TABLE 3 shows the comparative study with the aforementioned models according to the different saliency metrics, on DHF1K and UFC-SPORT datasets (300/47) testing videos. It is worth mentioning that our model is very competitive in the two Datasets. The 3DSAL-weighted repeatedly appears in the best two scores, and exhibits the best score for certain metrics. Also, it is clear that deep learning approaches outperform classic hand-crafted video saliency methods.
DHF1K. The diversity of this Dataset makes the prediction task very challenging, our model remains very competitive since our scores are close to the state of art model (ACLNet (Wang et al., 2018)). This is due to the inclusion of temporal domain exploration via the use 3D CNN for adjacent frames.

UFC-SPORT. On the 47 test videos of UFCSPORT Dataset, our model gains a remarkable advantage against other models. This demonstrates the capacity of our model to predict task driven saliency, when observers are more likely to track the main object in the scene e.g. soccer player, horse rider, skateboarder, etc. Most UFC-SPORT fixations

are located on the human body zone.

The 3DSAL-weighted model outperforms the 3DSAL-base model in all situations for to the UFCSPORT dataset. 3DSAL-base faces the problem of a centered saliency in the middle and considering the same weight for all frames $(c[i]=1)$ confuses the model to predict saliency map in a highly correlated space, which increases the false positive rate. We solved this problem when using the tanh weighting function, which helped the $3 \mathrm{D}$ CNN learn more accurate relationships between the features of adjacent frames ( e.g. AUC-J: $0.8111 \rightarrow 0.8813$, NSS: $1.7119 \rightarrow 2.8023$ ).

Fig 3 illustrates the prediction task on a sample of frames from three datasets: DHF1K, DAVIS, UFC-SPORT. It can be seen that the generated 


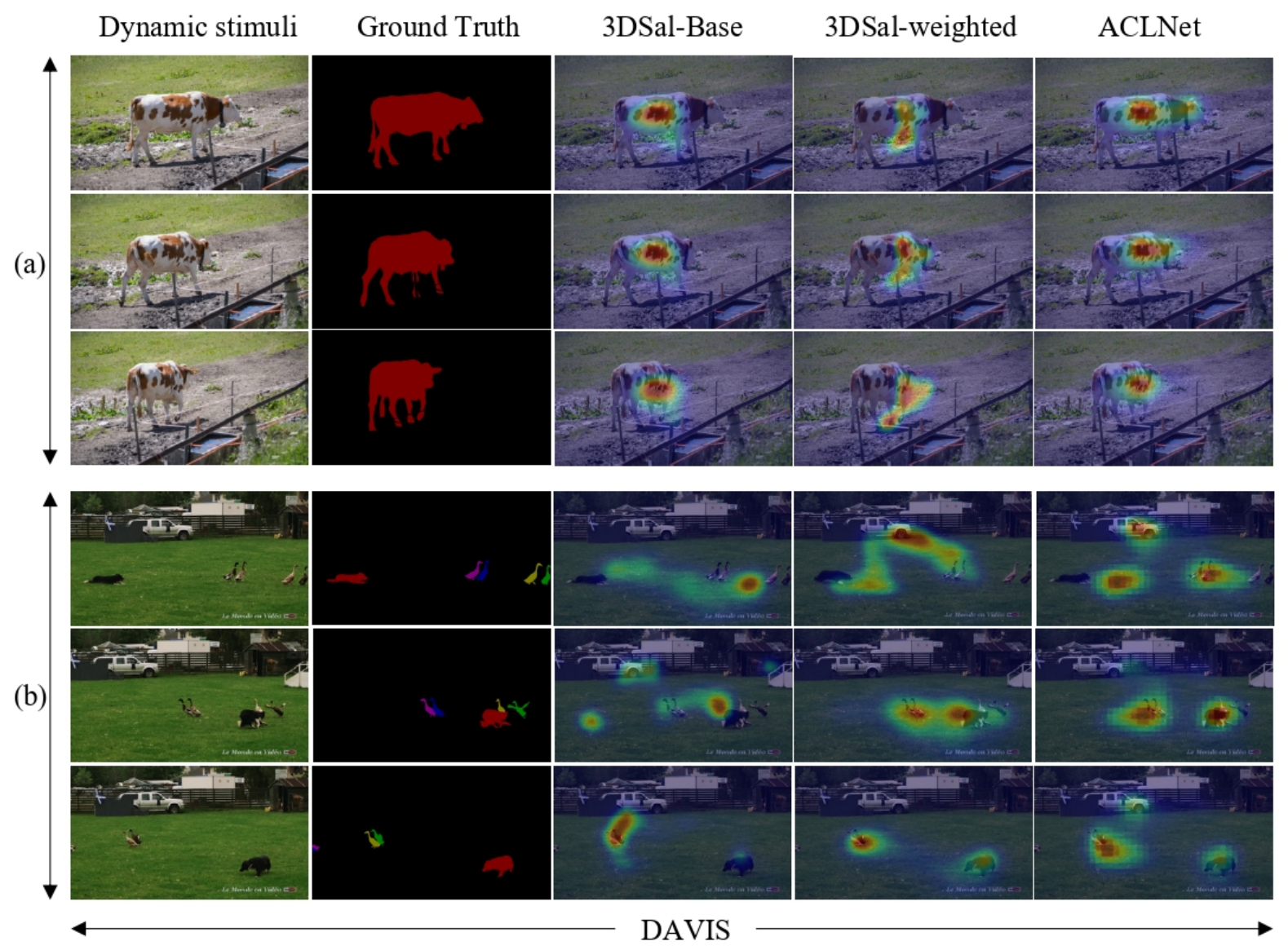

Figure 4: Qualitative results of our 3DSAL model and ACLNet (Wang et al., 2018) on two validation video samples from the object segmentation dataset DAVIS. It can be observed that the proposed 3DSAL-weighted is able to capture the main objects in the scene.

saliency maps with 3DSAL-weighted are more comprehensive and look remarkably similar to the Ground truth saliency maps in terms of fixations. DAVIS (Perazzi et al., 2016) is a video object segmentation dataset, thus, the various saliency metrics are not applicable. However, it is used in the qualitative study to show the effectiveness of our model to capture the main objects in the scene.

For more qualitative results, Fig 4 and Fig 5 show the ovelayed saliency maps on sample videos/frames from DAVIS and DHF1K datasets respectively, for the 3DSAL-Base, 3DSAL-weighted and ACLNet. Two main points could be derived from these figures:

- In Fig 5, it can be seen that the generated saliency maps using 3DSAL-base are sparse, this due to the big number of features in the latent space, the model tends to give a high probability to a given pixel, which makes it salient. In the 3DSALweighted version, the use of the weighting function forces the model to generate a more focused and consistent saliency regions.

- In Fig 4, it can be seen that, when the time goes by, the 3DSAL-weighted ignores some static objects and only focus on other moving objects, while ACLnet (Wang et al., 2018) still consider them as a salient regions. In video (b), both models considered the car as a salient object in the first frame. Since the car was static all over the scene, the 3DSAL-weighted considered it as a background, and only focused on dynamic objects (dog, ducks), while ACLNet (Wang et al., 2018) took it as salient during the whole scene. This demonstrates the effectiveness of 3D convolutions to capture motion.

\section{Conclusion}

In this paper, we target the problem of learning spatio-temporal features for video saliency pre- 


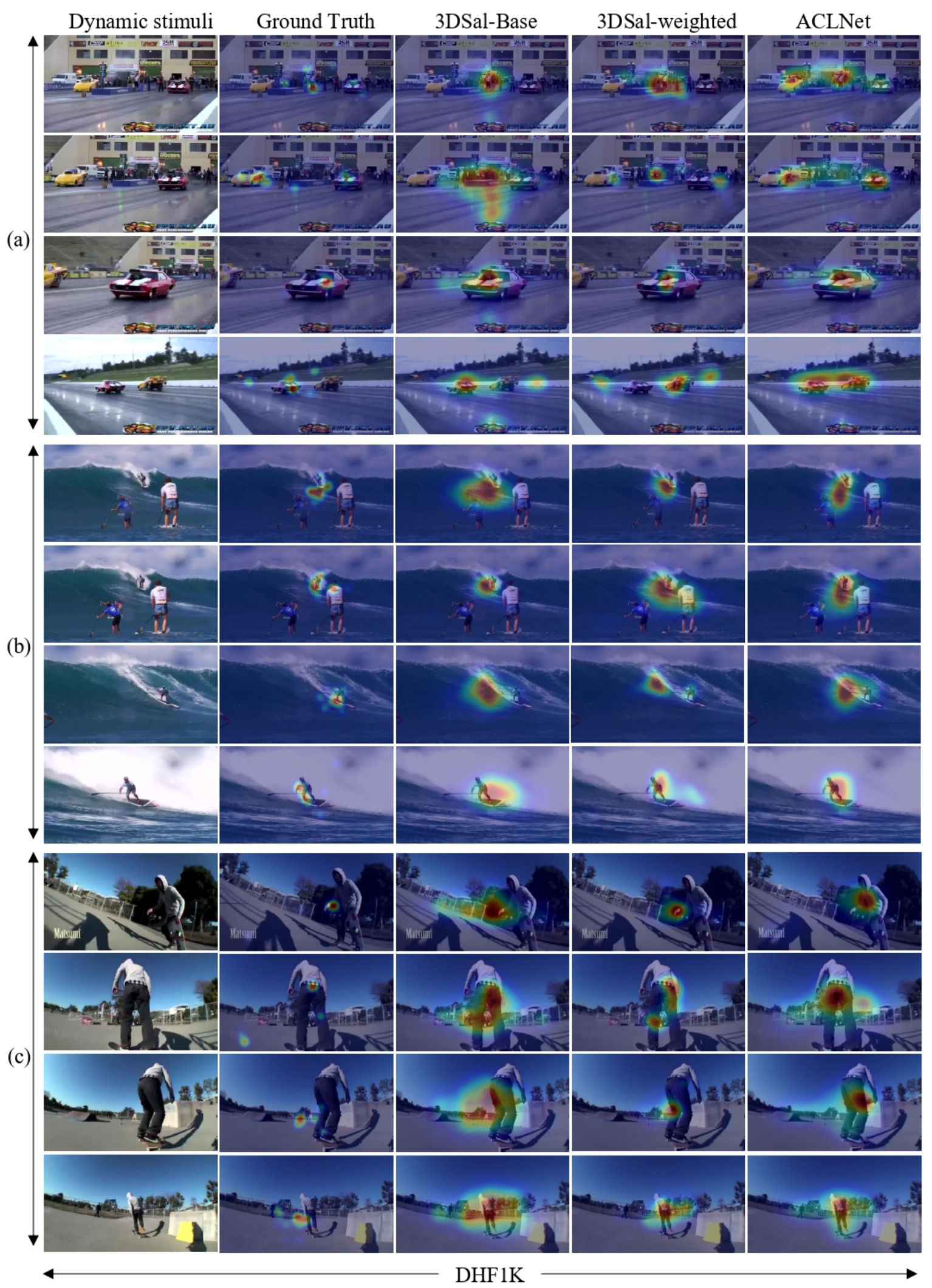

Figure 5: Qualitative results of our 3DSal model and ACLNet (Wang et al., 2018) on three validation video samples from DHF1K dataset. It can be observed that the proposed 3DSal-weighted is able to handle various challenging scenes well and produces consistent video saliency results. 
diction using 3D ConvNets, trained on large-scale video saliency datasets. We proposed the 3DSALweighted video saliency model, which fuses the spatio-temporal features from adjacent frames to accurately learn the hidden relationship that affects human behavior when watching videos. We extensively tested the performance of our model on: DHF1K, UFC-SPORT and DAVIS datasets, and reported the performance of our model compared with the stateof-the-art video saliency models. It is worth noting the competitiveness of the proposed model, whereby results on UFC-SPORT dataset outperform the stateof-the-art models.

\section{Acknowledgement}

The authors gratefully acknowledge the support of Ericsson Algeria for the donation of GPUs used in this work. This material is based on works supported by Science Foundation Ireland under Grant No. SFI/12/RC/2289 2 .

\section{REFERENCES}

Adel Bargal, S., Zunino, A., Kim, D., Zhang, J., Murino, V., and Sclaroff, S. (2018). Excitation backprop for rnns. In Proceedings of the IEEE Conference on Computer Vision and Pattern Recognition, pages 1440-1449.

Bak, C., Kocak, A., Erdem, E., and Erdem, A. (2018). Spatio-temporal saliency networks for dynamic saliency prediction. IEEE Transactions on Multimedia, 20(7):1688-1698.

Borji, A. (2018). Saliency prediction in the deep learning era: An empirical investigation. arXiv preprint arXiv:1810.03716.

Borji, A. and Itti, L. (2013). State-of-the-art in visual attention modeling. IEEE transactions on pattern analysis and machine intelligence, 35(1):185-207.

Borji, A. and Itti, L. (2015). Cat2000: A large scale fixation dataset for boosting saliency research. arXiv preprint arXiv:1505.03581.

Bruce, N. and Tsotsos, J. (2006). Saliency based on information maximization. In Advances in neural information processing systems, pages 155-162.

Bylinskii, Z., Judd, T., Borji, A., Itti, L., Durand, F., Oliva, A., and Torralba, A. Mit saliency benchmark.

Cornia, M., Baraldi, L., Serra, G., and Cucchiara, R. (2016). A deep multi-level network for saliency prediction. In 2016 23rd International Conference on Pattern Recognition (ICPR), pages 3488-3493. IEEE.

Deng, J., Dong, W., Socher, R., Li, L.-J., Li, K., and Fei-Fei, L. (2009). Imagenet: A large-scale hierarchical image database. In 2009 IEEE conference on computer vision and pattern recognition, pages 248-255. Ieee.
Gao, D. and Vasconcelos, N. (2005). Discriminant saliency for visual recognition from cluttered scenes. In Advances in neural information processing systems, pages 481-488.

Garcia-Diaz, A., Fdez-Vidal, X. R., Pardo, X. M., and Dosil, R. (2009). Decorrelation and distinctiveness provide with human-like saliency. In International Conference on Advanced Concepts for Intelligent Vision Systems, pages 343-354. Springer.

Garcia-Diaz, A., Fdez-Vidal, X. R., Pardo, X. M., and Dosil, R. (2012). Saliency from hierarchical adaptation through decorrelation and variance normalization. Image and Vision Computing, 30(1):51-64.

Goferman, S., Zelnik-Manor, L., and Tal, A. (2012). Context-aware saliency detection. IEEE transactions on pattern analysis and machine intelligence, 34(10):1915-1926.

Goodfellow, I., Pouget-Abadie, J., Mirza, M., Xu, B., Warde-Farley, D., Ozair, S., Courville, A., and Bengio, Y. (2014). Generative adversarial nets. In Advances in neural information processing systems, pages 2672-2680.

Guo, C., Ma, Q., and Zhang, L. (2008). Spatio-temporal saliency detection using phase spectrum of quaternion fourier transform. In 2008 IEEE Conference on Computer Vision and Pattern Recognition, pages 1-8. IEEE.

Hochreiter, S. and Schmidhuber, J. (1997). Long short-term memory. Neural computation, 9(8):1735-1780.

Hossein Khatoonabadi, S., Vasconcelos, N., Bajic, I. V., and Shan, Y. (2015). How many bits does it take for a stimulus to be salient? In Proceedings of the IEEE Conference on Computer Vision and Pattern Recognition, pages 5501-5510.

Huang, X., Shen, C., Boix, X., and Zhao, Q. (2015). Salicon: Reducing the semantic gap in saliency prediction by adapting deep neural networks. In Proceedings of the IEEE International Conference on Computer Vision, pages 262-270.

Itti, L., Koch, C., and Niebur, E. (1998). A model of saliency-based visual attention for rapid scene analysis. IEEE Transactions on Pattern Analysis \& Machine Intelligence, (11):1254-1259.

Ji, S., Xu, W., Yang, M., and Yu, K. (2013). 3d convolutional neural networks for human action recognition. IEEE transactions on pattern analysis and machine intelligence, 35(1):221-231.

Jiang, L., Xu, M., Liu, T., Qiao, M., and Wang, Z. (2018a). Deepvs: A deep learning based video saliency prediction approach. In The European Conference on Computer Vision (ECCV).

Jiang, L., Xu, M., Liu, T., Qiao, M., and Wang, Z. (2018b). Deepvs: A deep learning based video saliency prediction approach. In Proceedings of the European Conference on Computer Vision (ECCV), pages 602617

Jiang, L., Xu, M., and Wang, Z. (2017). Predicting video saliency with object-to-motion cnn and two-layer convolutional lstm. arXiv preprint arXiv:1709.06316. 
Jiang, M., Huang, S., Duan, J., and Zhao, Q. (2015). Salicon: Saliency in context. In The IEEE Conference on Computer Vision and Pattern Recognition (CVPR).

Judd, T., Durand, F., and Torralba, A. (2012). A benchmark of computational models of saliency to predict human fixations.

Kingma, D. P. and Ba, J. (2014). Adam: A method for stochastic optimization. arXiv preprint arXiv: 1412.6980 .

Kruthiventi, S. S., Ayush, K., and Babu, R. V. (2017). Deepfix: A fully convolutional neural network for predicting human eye fixations. IEEE Transactions on Image Processing, 26(9):4446-4456.

Kümmerer, M., Theis, L., and Bethge, M. (2014). Deep gaze i: Boosting saliency prediction with feature maps trained on imagenet. arXiv preprint arXiv:1411.1045.

Leboran, V., Garcia-Diaz, A., Fdez-Vidal, X. R., and Pardo, X. M. (2017). Dynamic whitening saliency. IEEE transactions on pattern analysis and machine intelligence, 39(5):893-907.

Leifman, G., Rudoy, D., Swedish, T., Bayro-Corrochano, E., and Raskar, R. (2017). Learning gaze transitions from depth to improve video saliency estimation. In Proceedings of the IEEE International Conference on Computer Vision, pages 1698-1707.

Li, X., Zhao, L., Wei, L., Yang, M.-H., Wu, F., Zhuang, Y., Ling, H., and Wang, J. (2016). Deepsaliency: Multitask deep neural network model for salient object detection. IEEE Transactions on Image Processing, 25(8):3919-3930.

Liu, N., Han, J., Zhang, D., Wen, S., and Liu, T. (2015). Predicting eye fixations using convolutional neural networks. In Proceedings of the IEEE Conference on Computer Vision and Pattern Recognition, pages 362370.

Mancas, M., Ferrera, V. P., Riche, N., and Taylor, J. G. (2016). From Human Attention to Computational Attention, volume 2. Springer.

Mathe, S. and Sminchisescu, C. (2015). Actions in the eye: Dynamic gaze datasets and learnt saliency models for visual recognition. IEEE transactions on pattern analysis and machine intelligence, 37(7):1408-1424.

Mital, P. K., Smith, T. J., Hill, R. L., and Henderson, J. M. (2011). Clustering of gaze during dynamic scene viewing is predicted by motion. Cognitive Computation, 3(1):5-24.

Nwankpa, C., Ijomah, W., Gachagan, A., and Marshall, S. (2018). Activation functions: Comparison of trends in practice and research for deep learning. CoRR, abs/1811.03378.

Pan, J., Ferrer, C. C., McGuinness, K., O'Connor, N. E., Torres, J., Sayrol, E., and Giro-i Nieto, X. (2017). Salgan: Visual saliency prediction with generative adversarial networks. arXiv preprint arXiv:1701.01081.

Panagiotis Linardos, Eva Mohedano, M. C. C. G. X. G.-i.N. (2018). Temporal saliency adaptation in egocentric videos.

Perazzi, F., Pont-Tuset, J., McWilliams, B., Van Gool, L., Gross, M., and Sorkine-Hornung, A. (2016). A benchmark dataset and evaluation methodology for video object segmentation. In Proceedings of the IEEE Conference on Computer Vision and Pattern Recognition, pages 724-732.

Prechelt, L. (1998). Early stopping-but when? In Neural Networks: Tricks of the trade, pages 55-69. Springer.

Rudoy, D., Goldman, D. B., Shechtman, E., and ZelnikManor, L. (2013). Learning video saliency from human gaze using candidate selection. In Proceedings of the IEEE Conference on Computer Vision and Pattern Recognition, pages 1147-1154.

Saslow, M. (1967). Effects of components of displacementstep stimuli upon latency for saccadic eye movement. Josa, 57(8):1024-1029.

Seo, H. J. and Milanfar, P. (2009). Static and space-time visual saliency detection by self-resemblance. Journal of vision, 9(12):15-15.

Simonyan, K. and Zisserman, A. (2014). Very deep convolutional networks for large-scale image recognition. arXiv preprint arXiv: 1409.1556.

Tran, D., Bourdev, L., Fergus, R., Torresani, L., and Paluri, M. (2015). Learning spatiotemporal features with $3 d$ convolutional networks. In Proceedings of the IEEE international conference on computer vision, pages $4489-4497$.

Vig, E., Dorr, M., and Cox, D. (2014). Large-scale optimization of hierarchical features for saliency prediction in natural images. In Proceedings of the IEEE Conference on Computer Vision and Pattern Recognition, pages 2798-2805.

Wang, W., Shen, J., Guo, F., Cheng, M.-M., and Borji, A. (2018). Revisiting video saliency: A large-scale benchmark and a new model. In Proceedings of the IEEE Conference on Computer Vision and Pattern Recognition, pages 4894-4903.

Xu, M., Jiang, L., Sun, X., Ye, Z., and Wang, Z. (2017). Learning to detect video saliency with hevc features. IEEE Transactions on Image Processing, 26(1):369 385

Zhang, J. and Sclaroff, S. (2013). Saliency detection: A boolean map approach. In Proceedings of the IEEE international conference on computer vision, pages 153-160. 